\title{
Renal Ultrasound contributes to Fabry Disease Diagnosis
}

Rodrigo F.C. Azambuja Neves ${ }^{1,2}$, Patrícia Varela ${ }^{3}$, Danilo Euclides Fernandes ${ }^{1 *}$, Michelle T. P. Riguetti', Simone Geraldini' ${ }^{1}, G u s t a v o$ Ferreira da Mata' ${ }^{1}$ Carmen Mendes ${ }^{4}$, Ana Maria Martins ${ }^{4}$, João Bosco Pesquero ${ }^{3}$, Gianna Mastroianni Kirsztajn'

'Section of Glomerulopathies, Nephrology Division, Federal University of São Paulo, São Paulo, Brazil ${ }^{2}$ Radiology Service of Hospital do Rim, São Paulo, Brazil

${ }^{3}$ Department of Biophysics, Federal University of São Paulo, São Paulo, Brazil

${ }^{4}$ IGEIM (Innate Errors of Metabolism), Federal University of São Paulo, São Paulo, Brazil

\section{Article Info}

\section{Article Notes}

Received: August 10, 2020

Accepted: August 25, 2020

\section{*Correspondence:}

Dr. Danilo Euclides Fernandes, Section of Glomerulopathies,

Nephrology Division, Federal University of São Paulo, São

Paulo, Brazil; Telephone No: + 55(11)5576-4848 VOIP 2471;

Email: defernandes@unifesp.br

Dr. Gianna Mastroianni Kirsztajn, Section of

Glomerulopathies, Nephrology Division, Federal

University of São Paulo, São Paulo, Brazil; Telephone No:

+55(11)5576-4848 VOIP 2471;

Email: gm.kirsztajn@unifesp.br

(c) 2020 Fernandes DE. This article is distributed under the terms of the Creative Commons Attribution 4.0 International License.

\section{Keywords:}

Parapelvic cysts

Fabry disease

ultrasound

kidney disease

glomerular filtration rate

glomerulopathy

\section{ABSTRACT}

Introduction: Renal impairment in Fabry disease is widely known, but the occurrence of renal cysts as a manifestation of the disease should still be highlighted among radiologists and other physicians.

Objectives: To compare the presence of parapelvic cysts between Fabry disease and other causes of chronic kidney disease (CKD).

Methods: We evaluated demographic, clinical and laboratory data, as well as kidney ultrasound (US) findings in 91 patients with Fabry disease $(n=37)$ and different glomerulopathies $(n=54)$.

Results: Both groups were similar in age $(p=0.29)$, gender $(p=0.98)$ eGFR $(p=0.10)$ and CKD stages $(p=0.19)$. Presence of parapelvic cysts differed significantly between those two groups $(p<0.0001$ in right and left kidneys; Cohen's $h=1.15$ ). When present, diameters of the parapelvic cysts were similar. Both groups had signs of CKD such as corticomedullary undifferentiation. None of the mutations we found in GLA gene was associated with a higher prevalence of parapelvic cysts.

Conclusions: When compared to different glomerulopathies, parapelvic cysts were more frequently found in Fabry disease despite age, gender and stage of CKD. Parapelvic cysts on ultrasound can raise suspicion on Fabry disease in patients with kidney disease of unknown etiology especially in the context of a familial nephropathy.

\section{Introduction}

Fabry disease (FD) is a rare condition caused by $\alpha$-Galalactosidase A ( $\alpha$-Gal A) deficiency. It is a X-linked disease (OMIM \#301500) caused by mutations in the gene that encodes the lysosomal a-Gal A enzyme, which affects glycosphingolipid metabolism. As a result of this deficiency, globotriaosylceramide (Gb3) and other glycosphingolipids accumulate inside the cells, causing organic dysfunction such as kidney damage ${ }^{1}$.

Although the major clinical manifestations of FD are well established, it can take years between the onset of the symptoms and final diagnosis, which remains a concern for physicians, patients and their families ${ }^{1}$.

Enzymatic analyses screening applies for an initial diagnosis in men who have risk factors or family history of inborn errors of metabolism. Women, who can be heterozygous for such mutations, must be genotyped. In men, genotyping allows family tracing for $\mathrm{FD}^{2}$.

Renal impairment in FD is widely known, but the occurrence of 
renal cysts as a manifestation of the disease should still be highlighted among radiologists and other specialist physicians. Few reports stated relevance, frequency and morphological characteristics of those renal cysts ${ }^{3,4}$. Our study shows that kidney ultrasound contributes to Fabry disease diagnosis among other causes of chronic kidney disease (CKD).

\section{Methods}

This is a cross-sectional study from the outpatient services of the Federal University of São Paulo (UNIFESP). It was approved by the Ethics Committee of UNIFESP and written informed consent was obtained from all participants.

We recruited 91 patients with CKD: 37 patients with FD (study group) and 54 patients with glomerulopathies (control group), comparable in age and estimated glomerular filtration rate (eGFR). Inclusion criteria were genetic-proven FD (GLA gene mutations as shown in Table A, supplementary material) for study group and biopsyproven glomerulopathies for control group.

Demographic, clinical and laboratory data were

Table 1. Demographical, clinical and ultrasound characteristics.

\begin{tabular}{|c|c|c|c|}
\hline & Fabry $(n=37)$ & Control $(n=54)$ & p-value \\
\hline Female, $\mathrm{n}(\%)$ & $19(51.4)$ & $32(59.3)$ & $0.29^{a}$ \\
\hline Age, years, mean(SD) & $36.4(16.5)$ & $37.7(14.7)$ & $0.98^{b}$ \\
\hline eGFR, mL/min, mean (SD) & $97.8(37.8)$ & $81.0(40.8)$ & $0.10^{c}$ \\
\hline Serum creatinine, mg/dL, mean (SD) & $1.2(1.7)$ & $1.4(1.2)$ & $0.56^{b}$ \\
\hline \multicolumn{4}{|l|}{ CKD stages, n(\%) } \\
\hline 1 & $19(63.3)$ & $27(51.9)$ & \multirow{5}{*}{$0.19^{a}$} \\
\hline 2 & $4(13.3)$ & $10(19.2)$ & \\
\hline 3 & $4(13.3)$ & $5(9.6)$ & \\
\hline 4 & $0(0.0)$ & $8(15.4)$ & \\
\hline 5 & $1(3.3)$ & $2(3.8)$ & \\
\hline Cysts & $20(54.1)$ & $13(24.1)$ & $<0.01^{\mathrm{a}}$ \\
\hline Unilateral & $6(16.2)$ & $8(14.8)$ & $1.00^{\mathrm{a}}$ \\
\hline Bilateral & $14(37.8)$ & $5(9.3)$ & $<0.001^{\mathrm{a}}$ \\
\hline \multicolumn{4}{|l|}{ Right kidney } \\
\hline Longitudinal axis, cm, mean (SD) & $9.8(2.2)$ & $10.8(1.4)$ & $0.01^{c}$ \\
\hline Laterolateral, cm, mean (SD) & $4.6(1.1)$ & $4.6(0.8)$ & $0.84^{c}$ \\
\hline Anteroposterior, cm, mean (SD) & $7.5(17.1)$ & $6.0(7.1)$ & $0.29^{c}$ \\
\hline Cysts & $18(48.6)$ & $7(13.0)$ & $<0.01^{\mathrm{a}}$ \\
\hline Cortical, $\mathrm{n}(\%)$ & $4(10.8)$ & $5(9.3)$ & $1.00^{\mathrm{a}}$ \\
\hline Largest diameter, $\mathrm{cm}$, mean (SD) & $2.5(2.7)$ & $1.7(1.2)$ & $0.76^{b}$ \\
\hline Smallest, cm, mean (SD) & $1.8(1.8)$ & $1.3(1.0)$ & $0.31^{b}$ \\
\hline Parapelvic, $\mathrm{n}(\%)$ & $11(29.7)$ & $2(3.7)$ & $<0.0001^{\mathrm{a}}$ \\
\hline Largest diameter, cm, mean (SD) & $1.0(0.4)$ & $1.2(0.0)$ & $0.81^{b}$ \\
\hline Smallest, cm, mean (SD) & $0.8(0.2)$ & $0.9(0.4)$ & $0.81^{b}$ \\
\hline \multicolumn{4}{|l|}{ Left kidney } \\
\hline Longitudinal axis, cm, mean (SD) & $9.6(2.5)$ & $10.8(1.4)$ & $0.08^{c}$ \\
\hline Anteroposterior, cm, mean (SD) & $4.6(1.1)$ & $5.0(0.8)$ & $0.10^{c}$ \\
\hline Cysts & $23(62.2)$ & $11(20.4)$ & $<0.001^{a}$ \\
\hline Cortical, n(\%) & $5(13.5)$ & $5(9.3)$ & $0.76^{a}$ \\
\hline Largest diameter, cm, mean (SD) & $3.2(3.0)$ & $1.0(0.4)$ & $0.09^{b}$ \\
\hline Smallest, cm, mean (SD) & $2.5(2.7)$ & $0.9(0.4)$ & $0.12^{\mathrm{b}}$ \\
\hline Parapelvic, $\mathrm{n}(\%)$ & $17(45.9)$ & $6(11.1)$ & $<0.0001^{\mathrm{a}}$ \\
\hline Largest diameter, cm, mean (SD) & $1.1(0.5)$ & $1.7(1.2)$ & $0.17^{\mathrm{b}}$ \\
\hline Smallest, cm, mean (SD) & $0.8(0.2)$ & $1.2(1.0)$ & $0.10^{b}$ \\
\hline CKD US features*, n (\%) & $5(13.5)$ & $5(9.3)$ & $0.6^{\mathrm{b}}$ \\
\hline
\end{tabular}

aPearson Chi Square test

${ }^{\mathrm{b}} \mathrm{T}$-student test

'Mann-Whitney test

* No corticomedullary distinction

Abbreviations: CKD, chronic kidney disease; eGFR, estimated glomerular filtration ratio; $\mathrm{n}$, sample; SD, standard-deviation. 


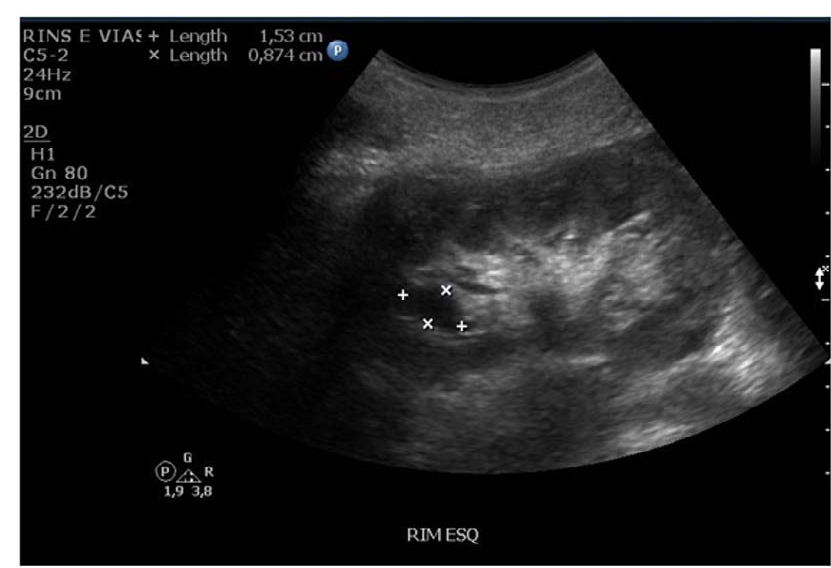

Figure 1. Ultrasound image of parapelvic renal cysts in a patient with Fabry disease.

obtained from the patients' chart. A 17-year specialist in Radiology performed a total abdomen ultrasound using a convex probe. The radiologist was blinded for the patient's diagnosis and determined renal diameters, presence of parapelvic (Figure 1) and/or cortical cysts, and corticomedullary differentiation (increase echogenicity). GLA mutations were investigated by Sanger sequencing as described by Varela et $\mathrm{al}^{5}$.

Data were typed and organized in Microsoft Excel for Mac $®$, version 16.35 , and analyzed in IBM $®$ SPSS statistics, version 26 . We used absolute and relative frequencies (nominal variables) and mean and standarddeviation (numeric variable) to describe our results. We compared the groups through Pearson Chi-Square and Mann-Whitney tests, as appropriate (alfa $=0.05$, confidence interval=95\%). Effect size were calculated according to Cohen's methods.

\section{Results}

We analyzed two groups of patients with CKD: 37 with FD and 54 with different glomerular diseases. They were similar in age $(p=0.24)$, gender $(p=0.79)$, eGFR ( $p$ $=0.11)$ and CKD stages $(p=0.06)$. In both groups, CKD stage 1 predominated $(66.7 \%$ of FD group and $51.9 \%$ of glomerulopathy group).

Control group accounted for minimal change disease (n $=3 ; 6 \%)$, membranous nephropathy $(\mathrm{n}=7 ; 13 \%)$, lupus nephritis ( $\mathrm{n}=14 ; 26 \%)$, IgA nephropathy ( $\mathrm{n}=5 ; 9 \%)$, pauci immune GN ( $\mathrm{n}=2 ; 3 \%)$, chronic GN $(\mathrm{n}=3 ; 6 \%)$, focal segmental glomerulosclerosis $(n=16 ; 30 \%)$, antiglomerular basement membrane $(\mathrm{n}=1 ; 2 \%)$ and Alport syndrome $(n=3 ; 6 \%)$.

The control group had slightly larger kidneys than the Fabry group. Presence of renal cysts differed significantly between those two groups in both right $(p<0.01)$ and left $(p<0.001)$ kidneys and parapelvic cysts were responsible for such difference ( $p<0.0001$ in right and left kidneys;
Cohen's $h=1.15)$. When present, diameters of the parapelvic cysts were similar. Both groups had signs of CKD such as corticomedullary undifferentiation. Extrarenal cysts were observed in two patients with FD (one with pancreatic cysts and other with liver cysts), but not in the control group.

FD patients had mutations on all seven exons, and intron 5 (Table A, supplementary material), and none of them was associated with the presence of parapelvic cysts.

\section{Discussion}

FD is a rare disease with high morbidity, which clinical picture is well known by physicians dealing with rare diseases, but it is widely underdiagnosed in daily practice. Diversified and unspecific signs and symptoms of the disease explain the difficulties in performing a clinical diagnosis or even raising suspicion of it.

Our results strongly support that renal ultrasound contributes to suspect of FD kidney involvement particularly when family history of kidney diseases are present, due to the higher frequency of parapelvic cysts in patients with FD. Fabry nephropathy is a glomerular disease and this is the reason why we chose a control group of other glomerulopathies. As cystic kidney diseases have unique and well-established findings in ultrasound, they can be easily diagnosed.

In our sample, there were individuals in all stages of CKD, with similar distribution, ensuring that both groups had patients with similar functional renal involvement. Stages 1 and 2 predominated in both groups, and more than half of patients were in stage 1 in both groups, as well. Thus, it was possible to rule out any eventual interference of impairment in GFR (particularly renal failure) on the development of cystic disease in the evaluated groups. It was also relevant that the groups had similar age distribution as it is well established that cortical cysts, for instance, are more frequent in older individuals ${ }^{3,4}$. The gender distribution was also similar, and this was important as FD has variable clinical pictures related to gender and a more exuberant clinical presentation is usually attributed to males ${ }^{7}$.

Our two groups differed only in terms of frequency of parapelvic cysts. All the other parameters - cortical cysts, renal volume, corticomedullary undifferentiation and lithiasis - seemed not to discriminate between FD and the evaluated glomerulopathies. Some diseases that typically present renal cysts may also show cysts in other organs. As some authors suggested6, we performed a complete abdominal ultrasound to identify extrarenal cysts, but they were uncommon in our sample.

Although this is a single-center study with a small sample, which prevent us from generalizing our results, 
we have a powerful sample to endorse that parapelvic cysts are more frequent in FD and may flag its diagnosis. Regardless of the expert sonographer in our study, nonexpert radiologists who perform ultrasound should be aware of findings that highly suggest a specific condition such as $\mathrm{FD}^{8-10}$ because renal ultrasound is commonly performed and plays an important role in the assessment of CKD. Renal ultrasound stands for a non-invasive, cheap and fast imaging method that is widely used in clinical practice. If present, parapelvic cysts could alert for this diagnosis and in the context of familial kidney disease be the earliest indication of FD considering the lack of specificity of most signs and symptoms of such disease.

\section{Conclusion}

Our findings endorse that parapelvic cysts on ultrasound can raise suspicion on FD, when compared to other renal diseases. Such cysts are more frequently found in FD despite age, gender and stage of CKD. If radiologists, nephrologists and other physicians are aware of such findings, the earlier diagnosis of FD may contribute to prevent loss of renal function among those patients.

\section{References}

1. Waldek, S. \& Feriozzi, S. Fabry nephropathy: a review - how can we optimize the management of Fabry nephropathy? BMC Nephrol. 15, 72 (2014).

2. Laney, D. A. et al. Fabry Disease Practice Guidelines: Recommendations of the National Society of Genetic Counselors. J. Genet. Couns. 22, 555564 (2013).

3. Pisani, A. et al. Parapelvic cysts, a distinguishing feature of renal Fabry disease. Nephrol. Dial. Transplant. Off. Publ. Eur. Dial. Transpl. Assoc. Eur. Ren. Assoc. 33, 318-323 (2018).

4. Ries, M. et al. Parapelvic kidney cysts: a distinguishing feature with high prevalence in Fabry disease. Kidney Int. 66, 978-982 (2004).

5. Varela, P. et al. Functional Characterization and Pharmacological Evaluation of a Novel GLA Missense Mutation Found in a Severely Affected Fabry Disease Family. Nephron 144, 147-155 (2020).

6. Vester, U., Kranz, B. \& Hoyer, P. F. The diagnostic value of ultrasound in cystic kidney diseases. Pediatr. Nephrol. 25, 231-240 (2010).

7. Del Pino, M. et al. Fabry Nephropathy: An Evidence-Based Narrative Review. Kidney Blood Press. Res. 43, 406-421 (2018).

8. Sayer, J. A., Haslam, P. \& Brennan, P. Parapelvic cysts leading to a diagnosis of Fabry disease. Kidney Int. 74, 1366 (2008).

9. Glass, R. B. J. et al. Fabry disease: renal sonographic and magnetic resonance imaging findings in affected males and carrier females with the classic and cardiac variant phenotypes. J. Comput. Assist. Tomogr. 28, 158-168 (2004).

10. McCloskey, S., Brennan, P. \& Sayer, J. A. Variable phenotypic presentations of renal involvement in Fabry disease: a case series. F1000Research 7, 356 (2018). 


\section{Supplementary Table:}

Table A. GLA gene mutations detected in the patients with Fabry disease submitted to ultrasound evaluation.

\begin{tabular}{|c|c|c|c|c|c|c|}
\hline $\mathbf{n}$ & Age (y) & Gender & Exon/Intron & Protein & Nucleotide & Classification \\
\hline 1 & 43 & $M$ & Exon 1 & p.(Trp24fsTer125) & c.59_72dupCCCTCGTTTCCTGG & Classic \\
\hline 2 & 41 & $\mathrm{~F}$ & Exon 7 & p.Arg356Trp & c. $1066 C>T$ & Late-onset \\
\hline 3 & 23 & $M$ & Exon 7 & p.Arg342GIn & c. $1025 \mathrm{G}>\mathrm{A}$ & Classic \\
\hline 4 & 59 & $\mathrm{~F}$ & Exon 1 & p.Cys63Tyr & c. $188 \mathrm{G}>\mathrm{A}$ & Classic \\
\hline 5 & 24 & M & Exon 1 & p.Cys63Tyr & c. $188 \mathrm{G}>\mathrm{A}$ & Classic \\
\hline 6 & 61 & $M$ & Exon 7 & p.Arg356Trp & c. $1066 C>T$ & Late-onset \\
\hline 7 & 47 & $M$ & Exon 1 & p.Cys52Ter & c. $156 C>A$ & Classic \\
\hline 8 & 20 & $\mathrm{~F}$ & Exon 1 & p.Cys52Ter & c. $156 C>A$ & Classic \\
\hline 9 & 17 & $M$ & Exon 1 & p.Cys52Ter & c. $156 C>A$ & Classic \\
\hline 10 & 58 & $M$ & Intron 5 & IVS5 c.802-2A>G & c. $802-2 A>G$ & Classic \\
\hline 11 & 65 & $\mathrm{~F}$ & Exon 7 & p.Arg342GIn & c. $1025 \mathrm{G}>\mathrm{A}$ & Classic \\
\hline 12 & 40 & $M$ & Exon 7 & p.Arg356Trp & c. $1066 C>T$ & Late-onset \\
\hline 13 & 62 & $\mathrm{~F}$ & Exon 6 & p.Met290lle & c. $870 G>A$ & Late-onset \\
\hline 14 & 44 & $\mathrm{~F}$ & Exon 1 & p.Asn34Asp & c. $100 A>G$ & Classic \\
\hline 15 & 9 & $M$ & Exon 1 & p.Arg49Pro & c. $146 G>C$ & Classic \\
\hline 16 & 48 & $\mathrm{~F}$ & Exon 1 & p.Arg49Pro & c. $146 \mathrm{G}>\mathrm{C}$ & Classic \\
\hline 17 & 31 & $\mathrm{~F}$ & Exon 1 & p.Cys52Phe & c.155G $>\mathrm{T}$ & GVUS \\
\hline 18 & 51 & $M$ & Exon 2 & p.Arg118Cys & c. $352 C>T$ & GVUS \\
\hline 19 & 27 & $\mathrm{~F}$ & Exons 2 and 6 & p.Phe113Leu / p.Asn313Tyr & c. $337 \mathrm{~T}>\mathrm{C} / \mathrm{c} .937 \mathrm{G}>\mathrm{T}$ & Late-onset / GVUS \\
\hline 20 & 50 & $\mathrm{~F}$ & Exon 2 & p.Phe113Leu & c. $337 \mathrm{~T}>\mathrm{C}$ & Late-onset \\
\hline 21 & 40 & $M$ & Exon 7 & p.Arg342Gln & c. $1025 \mathrm{G}>\mathrm{A}$ & Classic \\
\hline 22 & 34 & $\mathrm{~F}$ & Exon 1 & p.Cys52Ter & c. $156 C>A$ & Classic \\
\hline 23 & 28 & $\mathrm{~F}$ & Exon 5 & p.Arg220Ter & c. $658 \mathrm{C}>\mathrm{T}$ & Classic \\
\hline 24 & 17 & $\mathrm{~F}$ & Exon 1 & p.(Trp24fsTer125) & c.59_72dupCCCTCGTTTCCTGG & Classic \\
\hline 25 & 53 & $\mathrm{~F}$ & Exon 1 & p.(Trp24fsTer125) & c.59_72dupCCCTCGTTTCCTGG & Classic \\
\hline 26 & 17 & $\mathrm{~F}$ & Exon 1 & p.(Trp24fsTer125) & c.59_72dupCCCTCGTTTCCTGG & Classic \\
\hline 27 & 25 & $M$ & Exon 1 & p.Asn34Asp & c. $100 A>G$ & Classic \\
\hline 28 & 14 & $M$ & Exon 1 & p.Asn34Asp & c. $100 A>G$ & Classic \\
\hline 29 & 12 & M & Exon 1 & p.Asn34Asp & c. $100 A>G$ & Classic \\
\hline 30 & 18 & $M$ & Exon 7 & p.Arg342GIn & c. $1025 \mathrm{G}>\mathrm{A}$ & Classic \\
\hline 31 & 46 & $\mathrm{~F}$ & Exon 7 & p.Arg342GIn & c. $1025 \mathrm{G}>\mathrm{A}$ & Classic \\
\hline 32 & 23 & $M$ & Exon 7 & p.Arg356Trp & c. $1066 C>T$ & Late-onset \\
\hline 33 & 34 & $\mathrm{~F}$ & Exon 7 & p.Arg356Trp & c. $1066 C>T$ & Late-onset \\
\hline 34 & 38 & $\mathrm{~F}$ & Intron 5 & IVS5 c.802-2A>G & c. $802-2 A>G$ & Classic \\
\hline 35 & 16 & $\mathrm{~F}$ & Exon 7 & p.Arg356Trp & c. $1066 \mathrm{C}>\mathrm{T}$ & Late-onset \\
\hline 36 & 55 & $M$ & Exon 3 & p.Leu180Phe & c.540G>T & GVUS \\
\hline 37 & 53 & $\mathrm{~F}$ & Exon 1 & p.Cys52Ter & c. $156 C>A$ & Classic \\
\hline
\end{tabular}

Abbreviations: GVUS, genetic variants of unknown significance; $M$, male; $F$, female. 